\title{
Mannose-binding lectin plays a critical role in myocardial ischaemia and reperfusion injury in a mouse model of diabetes
}

\author{
M. N. Busche • M. C. Walsh • M. E. McMullen • \\ B. J. Guikema • G. L. Stahl
}

Received: 6 February 2008 / Accepted: 21 April 2008 / Published online: 21 May 2008

(C) Springer-Verlag 2008

\begin{abstract}
Aims/hypothesis Diabetic patients are at increased risk of cardiomyopathy, acute myocardial infarction and loss of cardiac progenitor cells (CPCs), but the aetiology is poorly understood. We hypothesised a significant role for mannose-binding lectin (MBL) in cardiomyopathies associated with hyperglycaemia.

Methods The role of MBL in myocardial ischaemia and reperfusion $(\mathrm{MI} / \mathrm{R})$ injury was investigated in wild-type (WT) and MBL-null mice following 2 weeks of streptozotocin-induced hyperglycaemia.

Results Hyperglycaemic WT mice presented with significantly decreased left ventricular ejection fractions and increased serum troponin I levels and myocardial inflammation compared with non-diabetic WT mice following MI/R. Hyperglycaemic MBL-null mice or insulin-treated diabetic WT mice were significantly protected from MI/R injury compared with diabetic WT mice. In an additional study using diabetic WT mice, echocardiographic measurements demonstrated signs of dilative cardiomyopathy, whereas heart:body weight ratios suggested hypertrophic cardiac remodelling after 2 weeks of hyperglycaemia. Immunohistochemical analysis of CPCs showed significantly lower numbers in diabetic WT hearts compared with non-diabetic hearts. Insulin-treated diabetic WT or untreated diabetic MBL-null mice were protected from dilative cardiomyopathy, hypertrophic remodelling and loss of CPCs.
\end{abstract}

M. N. Busche · M. C. Walsh • M. E. McMullen • B. J. Guikema •

G. L. Stahl $(\square)$

Center for Experimental Therapeutics and Reperfusion Injury,

Department of Anesthesiology, Perioperative and Pain Medicine,

Brigham and Women's Hospital, Harvard Medical School,

75 Francis Street, Thorn 705,

Boston, MA 02115, USA

e-mail: gstahl@zeus.bwh.harvard.edu
Conclusions/interpretation These data demonstrate that MBL may play a critical role in diabetic $M I / R$ injury. Further, the absence of MBL appears to inhibit hypertrophic remodelling and hyperglycaemia-induced loss of CPCs after just 2 weeks of hyperglycaemia in mice.

Keywords Animal · Complement .

Mannose-binding lectin · Type 1 diabetes

$\begin{array}{ll}\text { Abbreviations } \\ \mathrm{Ab} & \text { antibody } \\ \mathrm{CPC} & \text { cardiac progenitor cell } \\ \mathrm{EF} & \text { ejection fraction } \\ \mathrm{MBL} & \text { mannose-binding lectin } \\ \mathrm{MI} / \mathrm{R} & \text { myocardial ischaemia/reperfusion } \\ \mathrm{PMN} & \text { polymorphonuclear cell } \\ \mathrm{STZ} & \text { streptozotocin } \\ \text { WT } & \text { wild-type }\end{array}$

\section{Introduction}

The complement system plays a critical role in ischaemia/ reperfusion injury of the myocardium, muscle, ileum and kidney [1-6]. Three different complement pathways, the classic, lectin and alternative pathway, are recognised. The role of the three complement pathways in reperfusion injury has been controversial. Earlier studies stressed the importance of the classic pathway and natural antibodies [7-9], while more recent studies, including work from our laboratory, suggest that the lectin complement pathway is the major initiating pathway in myocardial ischaemia/ reperfusion $(\mathrm{MI} / \mathrm{R})$ injury $[2,10,11]$. A recent study by our group has demonstrated that mannose-binding lectin 
(MBL) interacts with IgM to activate the lectin complement pathway in vitro and in vivo [12]. Similar observations by others also support an antibody-dependent activation of the MBL-dependent lectin pathway in ischaemic conditions [13].

Ischaemia/reperfusion injury is often enhanced in hyperglycaemia or diabetes. Augmented MI/R injury in diabetic animals [14] suggests a significant role for reactive oxygen and nitrogen species $[15,16]$ and has linked oxidative stress to lectin complement pathway activation [10]. Other studies have linked oxidative stress in diabetes to a loss of cardiac progenitor cells (CPCs) and heart failure [17].

These laboratory data mirror clinical observations. Diabetic patients have increased incidences of atherosclerosis, coronary artery disease and myocardial infarction [18, 19]. Diabetic patients also have increased mortality, worse long-term prognosis after myocardial infarction and a higher risk of cardiomyopathy compared with non-diabetic patients [19-21]. Clinical studies suggest a role for the lectin complement pathway in diabetes-related renal complications [22]. Basic science studies using MBL-null mice and a streptozotocin (STZ)-induced type 1 diabetic model have uncovered a role for MBL in the development of diabetic kidney disease [23]. While there is an established role of the complement system in non-diabetic MI/R injury in animal models $[1,2,11]$, the importance of MBL in increasing $\mathrm{MI} / \mathrm{R}$ injury in diabetic vs non-diabetic models has not yet been evaluated. Therefore, we investigated the role of MBL in a STZ-induced hyperglycaemic mouse model undergoing $\mathrm{MI} / \mathrm{R}$ injury.

\section{Methods}

Preparation of diabetic mice and insulin treatment All animals used in $\mathrm{MI} / \mathrm{R}$ experiments were male mice 8 12 weeks old. C57BL/6 [wild-type (WT)] mice were obtained from Charles River Laboratories (Wilmington, MA, USA). MBL-null mice [24], backcrossed seven generations onto the C57BL/6 background, were used [2, 25]. The MBL-null mice were backcrossed while monitoring their genetic background using microsatellite analysis so that the mice are $96 \% \mathrm{C} 57 \mathrm{BL} / 6$. All procedures were reviewed and conducted in accordance with the Institute's Animal Care and Use Committee. Experiments were performed according to the standards and principles set forth in the Guide for the Care and Use of Laboratory Animals, as revised in 1996 (Institute of Laboratory Animal Research, Washington, DC, USA). Mice were made hyperglycaemic by a single injection of freshly prepared STZ solution $(200 \mathrm{mg} / \mathrm{kg}$ body weight in citrate saline, $\mathrm{pH} 4.2$, i.p.; Alexis, Lausanne, Switzerland) [26]. The mice were housed in cages of four mice each and had unlimited access to water and standard mouse chow. Three days after STZ injection and again immediately before the in vivo studies, the urinary glucose level was assessed with Glucostix (Diastix; Bayer, Elkhart, IN, USA). Mice with urinary glucose $>27.8 \mathrm{mmol} / \mathrm{l}$ in both tests were presumed to be hyperglycaemic. A group of STZ-injected mice was intensively treated with Lantus insulin (Sanofi-Aventis, Bridgewater, NJ, USA) at 2-6 IU, i.p. (based on daily urinary glucose levels) daily, starting on the third day after STZ injection to rule out any potential cardiotoxic effects of STZ.

Experimental $M I / R$ Experimental $\mathrm{MI} / \mathrm{R}$ was performed after 14 days of a hyperglycaemic state. The surgical procedure was similar to that previously published [2]. Briefly, diabetic and non-diabetic mice were intubated and ventilated and anaesthesia maintained with isoflurane. The chest was opened, a suture placed around the left anterior descending coronary artery and tightened. After 15 or $30 \mathrm{~min}$ of ischaemia, the ligation was loosened and the myocardium reperfused for $4 \mathrm{~h}$. An electrocardiogram (modified lead III) was evaluated before, during and after ischaemia and used to verify ischaemia and reperfusion.

Echocardiography Echocardiography was performed after mice had undergone experimental MI/R as described above to assess cardiac function. Previous work from our laboratory has shown that myocardial injury assessed by histological infarct analysis is directly correlated to loss of cardiac function as measured by echocardiography [2]. Echocardiography was performed $4 \mathrm{~h}$ after reperfusion using a Philips Sonos 5500 (Philips Medical Systems, Bothell, WA, USA) with a 7-12 MHz animal transducer (Agilent Technologies, Santa Clara, CA, USA), as we have described [2]. Ejection fraction (EF) was calculated from left ventricular M-mode measurements as well as $2 \mathrm{D}$ imaging from long-axis length and short-axis area measurements of the left ventricle $[27,28]$. For EF, only M-mode data are presented, as both methods of EF measurement produced identical results. Long-axis and short-axis area measurements of the left ventricle were assessed in an additional group of 16 animals (i.e. four mice per group) to assess myocardial dimensions under non-ischaemic conditions.

Collection of blood and tissue The chest cavity was opened, the inferior caval vein was cut and blood was collected from the thoracic cavity. Hearts were excised and fixed in PBS containing $10 \%\left(\mathrm{wt} / \mathrm{vol}\right.$.) formalin at $4{ }^{\circ} \mathrm{C}$ overnight. In 16 additional mice that did not undergo MI/R, heart and body weights were assessed in non-diabetic and diabetic animals at 10 weeks of age.

Serum troponin I concentrations Serum troponin I concentrations were measured as an index of myocardial cell death 
using a commercially available ELISA kit (Life Diagnostics, West Chester, PA, USA) [29].

Histological sections and staining for polymorphonuclear cells and CPCs Formalin-fixed hearts were dehydrated, embedded in paraffin and cut into $7 \mu \mathrm{m}$ sections. To evaluate polymorphonuclear cell (PMN) infiltration or CPCs in the heart, sections were dewaxed with EZ-DeWax Solution (BioGenex, San Ramon, CA, USA) and incubated with blocking buffer containing 5\% (vol./vol.) normal sheep (for PMNs) or donkey (for CPCs) serum for 1-2 $\mathrm{h}$ at room temperature. Primary antibody $(\mathrm{Ab})$ incubation for PMN identification was done with purified rat anti-mouse Ly-6G monoclonal Ab (BD Pharmingen, Franklin Lakes, NJ, USA) for $1-2 \mathrm{~h}$ at room temperature as previously described [30]. CPC localisation was done as described previously using purified goat anti-mouse SCF R/c-kit polyclonal Ab (R\&D Systems, Minneapolis, MN, USA) for $1-2 \mathrm{~h}$ at room temperature [17]. Following the primary $\mathrm{Ab}$ incubation, slides were washed four times for $15 \mathrm{~min}$ each with TBS-Tween and then incubated with a purified donkey anti-goat IRDye800 Ab or sheep anti-rat IRDye800 Ab (Rockland Immunochemicals, Gilbertsville, PA, USA) for 1-2 $\mathrm{h}$ at room temperature. After washing again four times for $15 \mathrm{~min}$ each, excess fluid was removed and slides covered with Gel Mount (Biomeda, Foster City, CA, USA) and cover slips (Fisher Scientific, Pittsburgh, PA, USA) and sealed after $1 \mathrm{~h}$ with clear nail polish. Finally, sections were scanned for PMN infiltration or CPCs with an Odyssey infrared imaging system (LI-COR, Lincoln, NE, USA) at $800 \mathrm{~nm}$.

Heart sections stained for PMNs or CPCs were also quantitatively analysed by pixel counting using ImageJ software (National Institutes of Health, Bethesda, MD, USA). To count cardiac PMNs or CPCs per high-powered field in fluorescence imaging, we used the staining protocol described above for the Odyssey infrared imaging system, except that an FITC-conjugated secondary Ab was used. PMNs or CPCs per field were counted by fluorescence imaging at $\times 100$ magnification using a Nikon Eclipse microscope (Nikon Instruments, Melville, NY, USA) in three different areas of each heart section for three mice per group.

Statistics All statistical analysis of data was performed using SigmaStat software (SPSS, Chicago, IL, USA). All data were evaluated using one-way ANOVA and post hoc analysis using the Student-Newman-Keuls method. All data are expressed as means \pm SE.

\section{Results}

Establishing a model of $M I / R$ injury in the diabetic mouse In the present study $30 \mathrm{~min}$ of ischaemia and $4 \mathrm{~h}$ of reperfusion in non-diabetic WT mice resulted in a significant decrease in left ventricular EF compared with sham-operated animals (Fig. 1). Increasing the ischaemic a

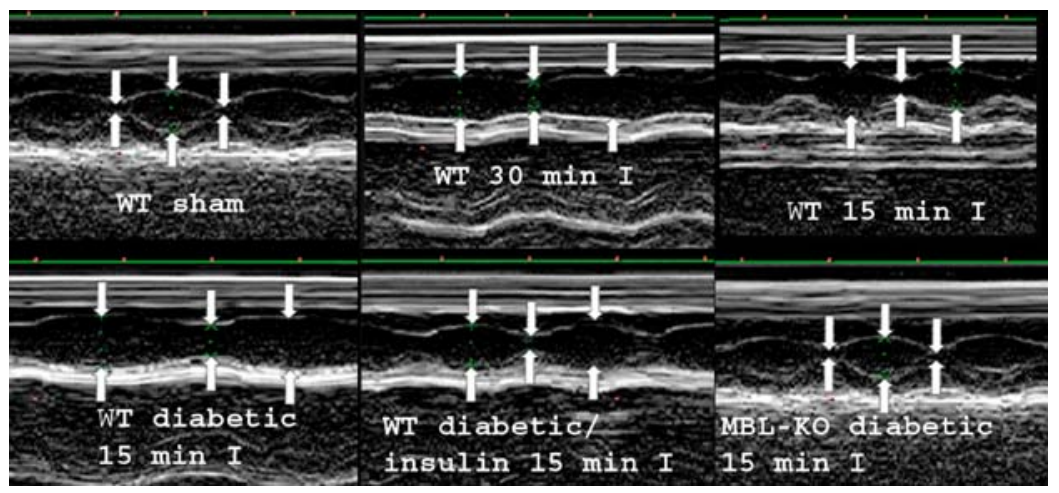

b

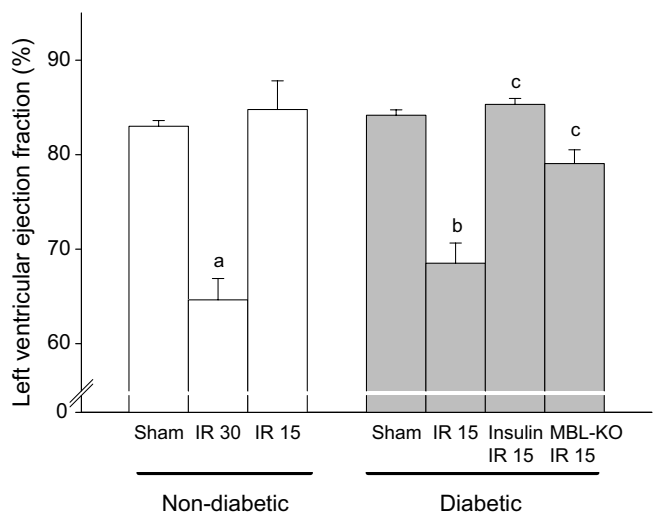

Fig. 1 Left ventricular function following MI/R. Echocardiography was performed and EF (\%) was calculated from the M-mode measurements as described in the Methods section. a Typical M-mode recordings. In the upper panels, non-diabetic WT mice that were shamoperated or underwent MI/R with 30 or $15 \mathrm{~min}$ of ischaemia and $4 \mathrm{~h}$ reperfusion (I). The lower panels represent diabetic animals after MI/R with $15 \mathrm{~min}$ of ischaemia (I) and $4 \mathrm{~h}$ of reperfusion: diabetic WT mice, insulin-treated diabetic WT mice and diabetic MBL-null (MBLKO) mice. b Summary of EF data. The left-hand group of bars represents non-diabetic WT mice that were sham-operated (sham) or underwent $\mathrm{MI} / \mathrm{R}$ with 30 and $15 \mathrm{~min}$ of ischaemia and $4 \mathrm{~h}$ of reperfusion (IR 30 and IR 15, respectively). The right-hand group of bars represents diabetic animals: sham-operated diabetic WT mice (sham), non-treated diabetic WT mice following MI/R (IR 15), insulin-treated diabetic WT mice following MI/R (insulin IR 15) and diabetic MBL-null mice after MI/R with $15 \mathrm{~min}$ of ischaemia and $4 \mathrm{~h}$ of reperfusion (MBL-KO IR 15). All data are means \pm SE of four to six animals per group. ${ }^{\mathrm{a}} p<0.001$ compared with non-diabetic WT sham and non-diabetic WT mice following MI/R with $15 \mathrm{~min}$ of ischaemia (IR 15). ${ }^{\mathrm{b}} p<0.001$ compared with diabetic WT sham, insulin-treated diabetic WT mice and diabetic MBL-null mice after MI/R with 15 min of ischaemia (IR 15). ${ }^{\mathrm{c}} p<0.001$ compared with diabetic WT mice following MI/R with 15 min of ischaemia (IR 15) 


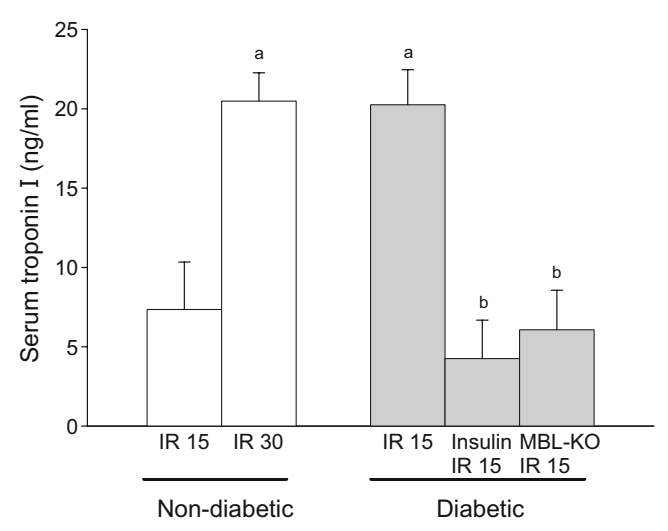

Fig. 2 Serum troponin I concentrations after MI/R. Serum troponin I concentrations after $\mathrm{MI} / \mathrm{R}$ were measured as described in the Methods section. The left group of bars represents non-diabetic WT mice that underwent $\mathrm{MI} / \mathrm{R}$ with 15 or $30 \mathrm{~min}$ of ischaemia and $4 \mathrm{~h}$ reperfusion (IR 15 and IR 30, respectively). The right group of bars represents diabetic animals that underwent $15 \mathrm{~min}$ of ischaemia and $4 \mathrm{~h}$ of reperfusion (IR): non-treated diabetic WT mice (IR 15), insulin-treated diabetic WT mice (Insulin IR 15) and non-treated diabetic MBL-null mice (MBL-KO IR 15). All data are means \pm SE of 4-6 animals per group. ${ }^{a} p<0.05$ compared with non-diabetic WT mice following MI/R with 15 min of ischaemia. ${ }^{\mathrm{b}} p<0.05$ compared with diabetic WT mice following $\mathrm{MI} / \mathrm{R}$ with $15 \mathrm{~min}$ of ischaemia

time to more than $30 \mathrm{~min}$ (i.e. up to $1 \mathrm{~h}$ ) increased mortality, but did not further decrease cardiac function as measured by echocardiography in surviving mice after $\mathrm{MI} / \mathrm{R}$ (data not shown). In non-diabetic WT mice, $15 \mathrm{~min}$ of ischaemia and $4 \mathrm{~h}$ of reperfusion do not induce a significant decrease in EF compared with sham-operated animals (Fig. 1a,b). In contrast to non-diabetic mice, MI/R in diabetic animals with $15 \mathrm{~min}$ of ischaemia induced a significant decrease in EF compared with sham-operated WT mice (Fig. 1a,b). Insulin treatment of diabetic WT mice prevented loss of cardiac function following MI/R (Fig. 1b). Induction of hyperglycaemia in MBL-null mice did not significantly alter EF following MI/R. The EF of sham-operated diabetic WT mice was comparable with sham-operated nondiabetic WT mice (Fig. 1b).

Diabetic MBL-deficient mice are protected from $M I / R$ induced increases in serum troponin I levels Thirty minutes of ischaemia and $4 \mathrm{~h}$ of reperfusion in non-diabetic WT mice significantly increased serum troponin I concentrations compared with only 15 min of ischaemia followed by reperfusion (Fig. 2, left side). In comparison with nondiabetic WT mice, diabetic WT mice had significantly increased serum troponin I concentrations following $\mathrm{MI} / \mathrm{R}$ (Fig. 2, right side). Serum troponin I concentrations after $\mathrm{MI} / \mathrm{R}$ were significantly reduced in insulin-treated WT diabetic animals compared with non-treated diabetic WT mice (Fig. 2). Diabetic MBL-null mice were significantly protected from cardiac injury following MI/R (Fig. 2).

Significantly decreased MI/R-related myocardial PMN infiltration in diabetic $M B L$-deficient mice In tissue samples from non-diabetic WT mice undergoing $15 \mathrm{~min}$ of ischaemia and $4 \mathrm{~h}$ of reperfusion, no significant PMN infiltration was observed (Fig. 3a,b). Compared with nondiabetic WT mice undergoing $15 \mathrm{~min}$ of ischaemia and $4 \mathrm{~h}$ of reperfusion, $30 \mathrm{~min}$ of ischaemia in non-diabetic WT mice significantly increased the inflammatory response a

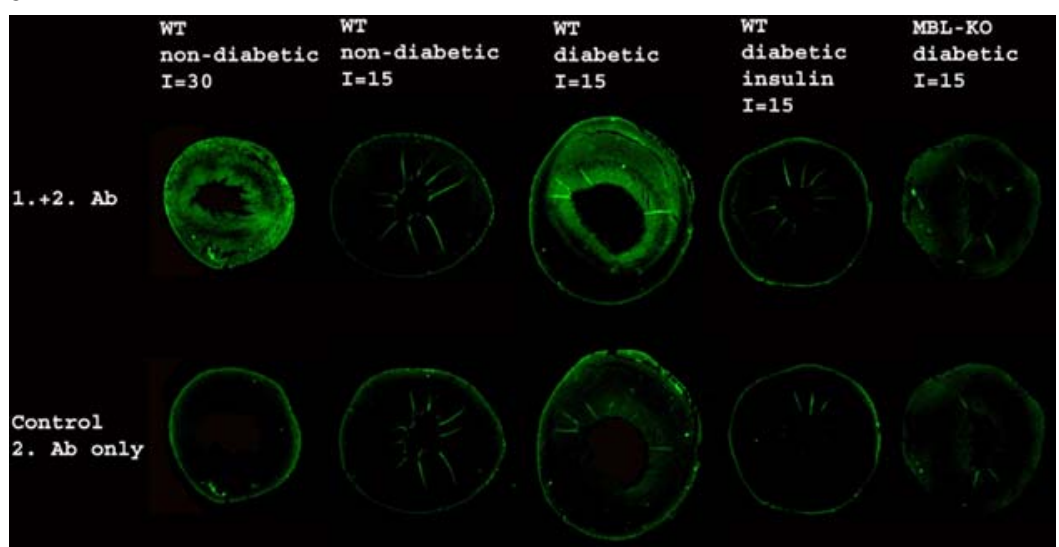

b

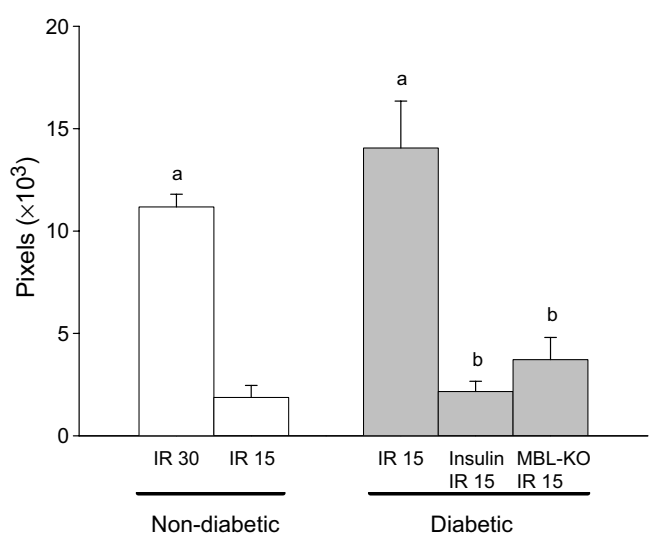

Fig. $3 \mathrm{PMN}$ infiltration into the myocardium following $\mathrm{MI} / \mathrm{R}$. a Representative sections. Heart sections were stained and PMN infiltration into the myocardium was measured using an infrared imaging system as described in the Methods section. Representative sections are shown from three animals per group. Upper row: $1 .+2$. $\mathrm{Ab}$ (primary and secondary $\mathrm{Ab}$ ). Lower row: control group with 2 . $\mathrm{Ab}$ only (secondary Ab only). Groups: non-diabetic WT mice following $\mathrm{MI} / \mathrm{R}$ with $30 \mathrm{~min}$ of ischaemia $(I=30)$ and $15 \mathrm{~min}$ of ischaemia $(I=15)$, respectively; diabetic WT mice following MI/R with $15 \mathrm{~min}$ of ischaemia untreated and insulin-treated, respectively; diabetic MBL-null mice (MBL-KO diabetic) following MI/R with $15 \mathrm{~min}$ of ischaemia. b Quantitative analysis of sections by pixel count. Pictures from heart sections stained for PMNs from three animals per group were quantitatively analysed by pixel counting using ImageJ software. ${ }^{\mathrm{a}} p<0.001$ compared with non-diabetic WT mice following MI/R with 15 min of ischaemia. ${ }^{\mathrm{b}} p<0.001$ compared with diabetic WT mice following MI/R with 15 min of ischaemia 


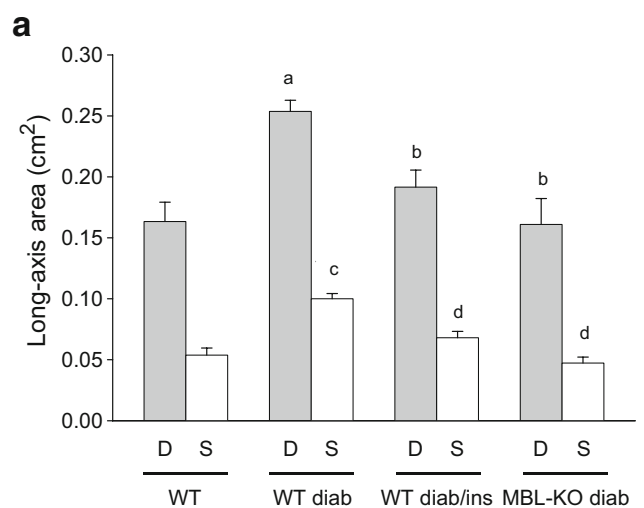

Fig. 4 Left ventricular area measurements. a Long-axis area measurements. Long-axis diastolic (D) and systolic (S) areas $\left(\mathrm{cm}^{2}\right)$ were measured in non-diabetic (WT) mice, diabetic WT (WT diab) mice after 2 weeks of diabetes, insulin-treated diabetic WT (WT diab/ins) mice and diabetic MBL-null (MBL-KO diab) mice. All data are means \pm SE of four animals per group. ${ }^{a} p<0.05$ compared with diastolic areas of non-diabetic WT mice. ${ }^{b} p<0.05$ compared with diastolic areas of non-treated diabetic WT mice. ${ }^{\mathrm{c}} p<0.001$ compared with systolic areas of non-diabetic WT mice. ${ }^{d} p<0.05$ compared with systolic areas of non-treated diabetic WT mice. b Short-axis area

(Fig. 3a,b). When diabetic WT mice were subjected to $15 \mathrm{~min}$ of ischaemia and $4 \mathrm{~h}$ of reperfusion, a significant increase in the inflammatory response was observed compared with non-diabetic WT mice undergoing MI/R with $15 \mathrm{~min}$ of ischaemia. The inflammatory response in the diabetic WT mouse was similar to that observed following $30 \mathrm{~min}$ of ischaemia in non-diabetic WT mice (Fig. 3a,b). Treatment of diabetic WT mice with insulin significantly attenuated the MI/R-induced inflammatory response. Diabetic MBL-null mice showed significantly reduced inflammatory infiltrates compared with diabetic WT mice (Fig. 3a,b).

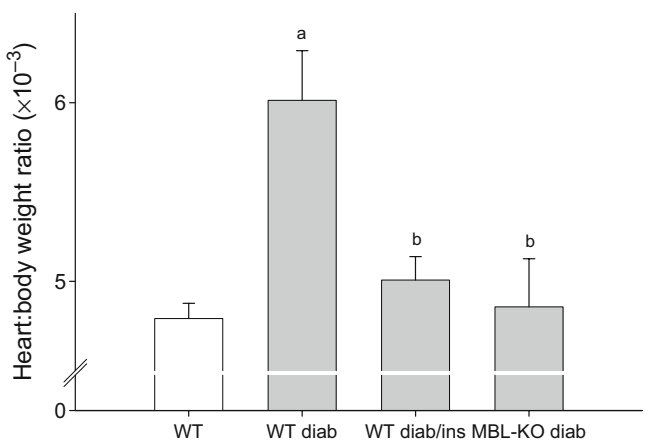

Fig. 5 Heart:body weight ratios. In 16 additional mice that did not undergo $\mathrm{MI} / \mathrm{R}$, heart and body weight values were obtained in nondiabetic and diabetic animals at 10 weeks of age. In diabetic animals, diabetes was induced at 8 weeks of age. Heart:body weight ratios were determined. All data are means $\pm \mathrm{SE}$ of four animals per group. ${ }^{\mathrm{a}} p<0.05$ compared with non-diabetic WT mice. ${ }^{\mathrm{b}} p<0.05$ compared with diabetic WT mice. diab/ins, diabetes and insulin-treated; MBL-KO diab, MBL-null mice made diabetic

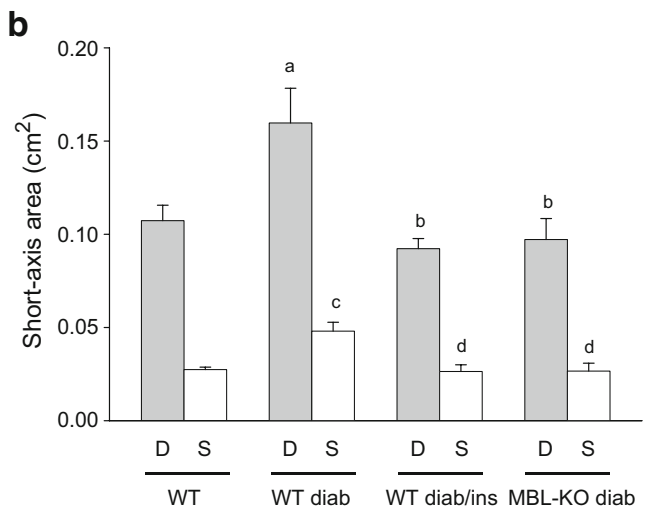

measurements. Short-axis diastolic (D) and systolic (S) areas $\left(\mathrm{cm}^{2}\right)$ were measured in non-diabetic WT (WT) mice, diabetic WT (WT diab) mice after 2 weeks of diabetes, insulin-treated diabetic WT (WT diab/ins) mice and diabetic MBL-null (MBL-KO diab) mice. All data are means $\pm \mathrm{SE}$ of four animals per group. ${ }^{\mathrm{a}} p<0.05$ compared with diastolic areas of non-diabetic WT mice. ${ }^{\mathrm{b}} p<0.05$ compared with diastolic areas of non-treated diabetic WT mice. ${ }^{c} p<0.05$ compared with systolic areas of non-diabetic WT mice. ${ }^{\mathrm{d}} p<0.05$ compared with systolic areas of non-treated diabetic WT mice

Hand counting PMNs in cardiac tissue demonstrated that non-diabetic WT mice undergoing MI/R with $30 \mathrm{~min}$ of ischaemia had a significant increase in the inflammatory response compared with non-diabetic WT mice undergoing $\mathrm{MI} / \mathrm{R}$ with $15 \mathrm{~min}$ of ischaemia, with $20 \pm 1$ vs $5 \pm 1$ $(p<0.001)$ PMNs per high-powered field, respectively. Diabetic WT mice undergoing MI/R with 15 min of ischaemia presented significantly increased PMN infiltration into the myocardium compared with non-diabetic WT mice undergoing the same amount of ischaemia with $27 \pm 1$ vs $5 \pm 1$ $(p<0.001)$ PMNs per high-powered field. Insulin-treated diabetic WT mice and non-treated diabetic MBL-null mice showed significantly reduced inflammatory infiltrates compared with diabetic WT mice with $4 \pm 1$ and $5 \pm 1$ vs $27 \pm 1$ PMNs per high-powered field $(p<0.001)$, respectively.

Echocardiographic evidence of dilative cardiomyopathy in diabetic mice After 2 weeks of hyperglycaemia, WT mice demonstrated significantly increased long- and short-axis area measurements during systole and diastole by echocardiography compared with non-diabetic animals (Fig. 4a,b). Additionally, insulin treatment protected diabetic WT mice from diabetic cardiomyopathy as measured from long- and short-axis measurements by echocardiography (Fig. 4a,b). Diabetic MBL-null mice were also protected from diabetesinduced changes in myocardial long- and short-axis measurements.

Heart:body weight ratios suggest diabetic hypertrophic cardiac remodelling Heart:body weight ratios after 2 weeks of hyperglycaemia were significantly increased compared 
a

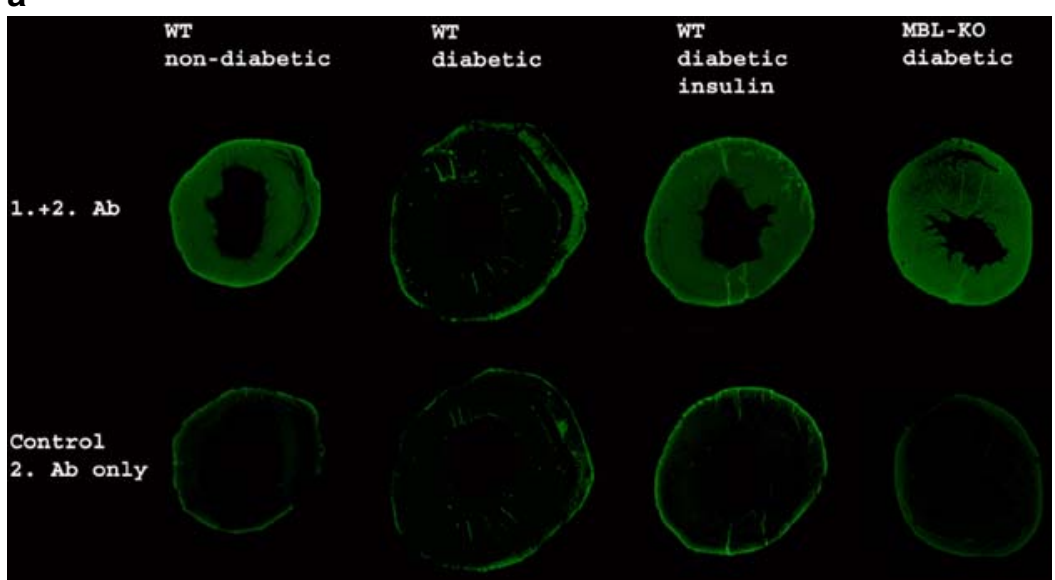

b

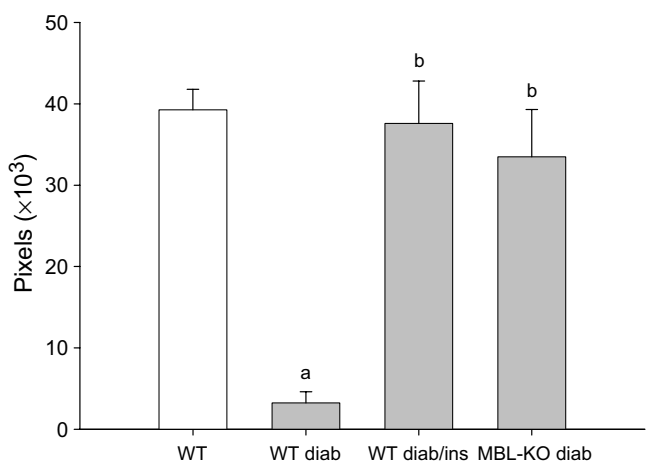

Fig. 6 CPC numbers. a Representative sections. Heart sections were stained and CPC numbers were measured using an infrared imaging system as described in the Methods. Representative sections are shown from three animals per group. Upper row: 1. + 2. Ab (primary and secondary $\mathrm{Ab}$ ). Lower row: control group with 2. $\mathrm{Ab}$ only (secondary $\mathrm{Ab}$ only). Groups: non-diabetic WT mice; diabetic WT mice following 2 weeks of diabetes untreated and insulin-treated, respectively; diabetic MBL-null (MBL-KO) mice following 2 weeks of diabetes. b Quantitative analysis of sections by pixel count. Pictures from heart sections stained for CPCs from three animals per group were quantitatively analysed by pixel counting using ImageJ software. Means \pm SE. ${ }^{a} p<0.001$ compared with non-diabetic WT mice following MI/R with 15 min of ischaemia. ${ }^{b} p<0.001$ compared with diabetic WT mice following MI/R with 15 min of ischaemia with non-diabetic WT mice (Fig. 5). Insulin treatment of diabetic WT mice significantly inhibited this increased ratio. Diabetic MBL-null mice did not demonstrate an increase in the heart:body weight ratio.

Diabetic MBL-deficient mice are protected from loss of CPCs staining for CPCs showed significantly lower numbers of CPCs in diabetic WT mice compared with non-diabetic WT mice (Fig. 6a,b). Insulin treatment prevented CPC loss in diabetic WT mice. Diabetic MBLnull mice were significantly protected from CPC loss (Fig. 6). CPC counts per high-powered field were also performed. Diabetic WT mice had significantly lower CPC numbers compared with non-diabetic controls with $4 \pm 1$ vs $17 \pm 2$ CPC per high-powered field $(p<0.001)$. Insulintreated diabetic WT mice and non-treated diabetic MBLnull mice were significantly protected from CPC loss compared with diabetic WT mice with $16 \pm 1$ and $15 \pm 2$ vs $4 \pm 1$ CPC per high-powered field $(p<0.001)$, respectively.

\section{Discussion}

Several significant observations were made in this study. First, we demonstrated a significant increase in myocardial injury in diabetic compared with non-diabetic mice following MI/R. Second, MBL-null mice were protected from myocardial injury following MI/R. Third, following 2 weeks of hyperglycaemia, we observed myocardial remodelling, which was inhibited by insulin treatment in WT mice or by the absence of MBL. These observations suggest that prevention of hyperglycaemia with insulin or the lack of MBL prevents the cardiomyopathies observed in this model of type 1 diabetic mouse.

We have previously demonstrated that this model of murine $\mathrm{MI} / \mathrm{R}$ injury is associated with increased inflammation, cardiac dysfunction (i.e. decreased EF) and complement deposition compared with sham-operated mice [2]. Similarly, in the present study $30 \mathrm{~min}$ of ischaemia and $4 \mathrm{~h}$ of reperfusion in non-diabetic WT mice resulted in a significant decrease in left ventricular EF, inflammation and myocardial injury compared with sham-operated animals. Increasing the ischaemic time to $60 \mathrm{~min}$ did not result in increased injury, but did result in increased mortality. Thus we decreased the ischaemic time to $15 \mathrm{~min}$ in our hyperglycaemia study, so that we could observe any increase in hyperglycaemia-induced injury following MI/R. Hyperglycaemia increased tissue injury (troponin I; Fig. 2), decreased cardiac function (EF; Fig. 1) and increased inflammation (PMN staining; Fig. 3) following $\mathrm{MI} / \mathrm{R}$ compared with the non-diabetic state and support the findings of others [14]. Troponin I concentrations and PMN infiltration into the myocardium mirrored the echocardiographic results. Furthermore, hyperglycaemic MBL-null mice were protected from $\mathrm{MI} / \mathrm{R}$ injury and alterations in the measured endpoints. Thus, hyperglycaemia for only 2 weeks results in enhanced cardiac abnormalities associated with $\mathrm{MI} / \mathrm{R}$, which can be significantly attenuated by the absence of MBL.

Earlier studies suggested that cardiotoxic effects of STZ are caused by insulin deficiency and not to a primary cardiotoxic effect of STZ [31]. Supporting these results, 
insulin treatment in our study attenuated the myocardial injury and remodelling observed in STZ-induced diabetes, ruling out a significant cardiotoxic effect of STZ.

When we were developing the new staining technique for quantifying murine myocardial inflammation using the Odyssey system, we observed that the diabetic WT myocardium was significantly larger than that of the nondiabetic WT mouse (see Fig. 3a). We then performed another set of studies to investigate this subjective observation in a group of mice that were not subjected to MI/R. Diabetic WT mice had significantly increased longand short-axis areas during systole and diastole compared with non-diabetic animals (Fig. 4). Furthermore, heart:body weight ratios were increased in diabetic WT compared with non-diabetic WT, diabetic MBL-null or insulin-treated diabetic WT mice. In a similar model of type 1 diabetes, a significant increase in kidney weight was observed in diabetic WT mice compared with diabetic MBL-null mice [23]. These data suggest that short periods of hyperglycaemia in a type 1 model of diabetes induce remodelling resulting in hypertrophic tissues that is inhibited by insulin treatment or the absence of MBL.

Enhanced oxidative stress in STZ-induced diabetes has been linked to CPC loss, thus impairing myocyte regeneration [17]. Our study demonstrates and confirms that diabetic WT mice lose significant CPC numbers following 2 weeks of diabetes. Diabetic MBL-null mice were protected from CPC loss, suggesting a significant role for this innate immune molecule. Oxidative stress has been shown to induce lectin pathway activation by increasing MBL binding [32, 33]. Thus, hyperglycaemia may induce myocardial oxidative stress, resulting in more MBL binding and lectin complement pathway activation. However, we have not fully investigated the potential role of lectin complement pathway activation in this model and this is a subject of present investigation. The CPC loss in diabetic WT hearts may play an important role in hypertrophic remodelling and increased susceptibility to MI/R in type 1 diabetes.

Limitations to the present study MBL deficiency in humans is common and depending on definition, more than $10 \%$ of the general population may be classified as MBL deficient [34]. We do not know if the results obtained in these murine studies will translate to the clinic. Future studies with established sample repositories and patient databases are clearly warranted. Further, while type 1 diabetes is a significant disease, type 2 diabetes is a growing health concern associated with various cardiovascular problems. Whether similar results will be observed in type 2 diabetes is unknown at the present time, but studies are underway. Further, we acknowledge that the length of hyperglycaemia used in this study is very short compared with the clinical setting. Future studies using other models of diabetes are needed to investigate the length and dose-responsiveness to varying degrees of hyperglycaemia.

In conclusion, our study demonstrates a pathophysiological role for MBL in hyperglycaemic CPC loss, myocardial remodelling and $\mathrm{MI} / \mathrm{R}$ injury. Our findings, together with findings from others [23], suggest that MBL plays an important role in type 1 diabetes-induced tissue injury. Further studies are needed to investigate whether similar observations are present in type 2 diabetes and whether these findings translate to the clinic.

Acknowledgements We acknowledge the expert technical assistance of L. Schaffer and M. Morrissey during the course of these studies. We thank K. Takahashi for providing the MBL-null breeding pairs. We thank S. K. Shernan for his expert advice on echocardiography. M. N. Busche received a research fellowship from the Deutsche Forschungsgemeinschaft (DFG, German Research Foundation). This work was supported in part by NIH grants HL56068, HL52886, HL79758, DE016191 and DE017821. This study was presented in part at the 11th European Meeting on Complement in Human Disease.

Duality of interest The authors declare that there is no duality of interest associated with this manuscript.

\section{References}

1. Weisman HF, Bartow T, Leppo MK et al (1990) Soluble human complement receptor type 1: in vivo inhibitor of complement suppressing post-ischemic myocardial inflammation and necrosis. Science 249:146-151

2. Walsh MC, Bourcier T, Takahashi K et al (2005) Mannosebinding lectin is a regulator of inflammation that accompanies myocardial ischemia and reperfusion injury. J Immunol 175:541-546

3. Hill JH, Ward PA (1971) The phlogistic role of C3 leukotactic fragments in myocardial infarcts in rats. J Exp Med 133:885-900

4. Pemberton M, Anderson G, Vetvicka V, Justus DE, Ross GD (1993) Microvascular effects of complement blockade with soluble recombinant $\mathrm{CR} 1$ on ischemia/reperfusion injury of skeletal injury. J Immunol 150:5104-5113

5. Wada K, Montalto MC, Stahl GL (2001) Inhibition of complement C5 reduces local and remote organ injury after intestinal ischemia/reperfusion in the rat. Gastroenterology 120:126-133

6. Stahl GL, Xu Y, Hao L et al (2003) Role for the alternative complement pathway in ischemia/reperfusion injury. Am J Pathol $162: 449-455$

7. Buerke M, Murohara T, Lefer AM (1995) Cardioprotective effects of a $\mathrm{C} 1$ esterase inhibitor in myocardial ischemia and reperfusion. Circulation 91:393-402

8. Horstick G, Heimann A, Gotze O et al (1997) Intracoronary application of $\mathrm{C} 1$ esterase inhibitor improves cardiac function and reduces myocardial necrosis in an experimental model of ischemia and reperfusion. Circulation 95:701-708

9. Zhang M, Austen WG Jr, Chiu I et al (2004) Identification of a specific self-reactive IgM antibody that initiates intestinal ischemia/reperfusion injury. Proc Natl Acad Sci USA 101:3886-3891

10. Collard CD, Vakeva A, Morrissey MA et al (2000) Complement activation following oxidative stress: Role of the lectin complement pathway. Am J Pathol 156:1549-1556 
11. Jordan JE, Montalto MC, Stahl GL (2001) Inhibition of mannosebinding lectin reduces postischemic myocardial reperfusion injury. Circulation 104:1413-1418

12. McMullen ME, Hart ML, Walsh MC, Buras J, Takahashi K, Stahl GL (2006) Mannose-binding lectin binds IgM to activate the lectin complement pathway in vitro and in vivo. Immunobiology 211:759-766

13. Zhang M, Takahashi K, Alicot EM et al (2006) Activation of the lectin pathway by natural IgM in a model of ischemia/reperfusion injury. J Immunol 177:4727-4734

14. Greer JJ, Ware DP, Lefer DJ (2006) Myocardial infarction and heart failure in the $\mathrm{db} / \mathrm{db}$ diabetic mouse. Am J Physiol Heart Circ Physiol 290:H146-H153

15. Dandona $\mathrm{P}$, Thusu K, Cook $\mathrm{S}$ et al (1996) Oxidative damage to DNA in diabetes mellitus. Lancet 347:444-445

16. Pozo-Navas B, Stessel H, Wolkart G, Brunner F (2006) Role of myocardial nitric oxide in diabetic ischemia-reperfusion dysfunction: studies in mice with myocyte-specific overexpression of endothelial nitric-oxide synthase. J Pharmacol Exp Ther 319:729-738

17. Rota M, LeCapitaine N, Hosoda T et al (2006) Diabetes promotes cardiac stem cell aging and heart failure, which are prevented by deletion of the p66shc gene. Circ Res 99:42-52

18. Haffner SM, Lehto S, Ronnemaa T, Pyorala K, Laakso M (1998) Mortality from coronary heart disease in subjects with type 2 diabetes and in nondiabetic subjects with and without prior myocardial infarction. N Engl J Med 339:229-234

19. Jacobs J, Sena M, Fox N (1991) The cost of hospitalization for the late complications of diabetes in the United States. Diabet Med 8 (Spec No.):S23-S29

20. Malmberg K, Yusuf S, Gerstein HC et al (2000) Impact of diabetes on long-term prognosis in patients with unstable angina and non-Q-wave myocardial infarction: results of the OASIS (Organization to Assess Strategies for Ischemic Syndromes) Registry. Circulation 102:1014-1019

21. Jelesoff NE, Feinglos M, Granger CB, Califf RM (1996) Outcomes of diabetic patients following acute myocardial infarction: a review of the major thrombolytic trials. Coron Artery Dis 7:732-743

22. Hovind P, Hansen TK, Tarnow L et al (2005) Mannose-binding lectin as a predictor of microalbuminuria in type 1 diabetes: an inception cohort study. Diabetes 54:1523-1527
23. Ostergaard J, Thiel S, Gadjeva M, Hansen TK, Rasch R, Flyvbjerg A (2007) Mannose-binding lectin deficiency attenuates renal changes in a streptozotocin-induced model of type 1 diabetes in mice. Diabetologia 50:1541-1549

24. Shi L, Takahashi K, Dundee J et al (2004) Mannose-binding lectin-deficient mice are susceptible to infection with Staphylococcus aureus. J Exp Med 199:1379-1390

25. Hart ML, Ceonzo KA, Shaffer LA et al (2005) Gastrointestinal ischemia-reperfusion injury is lectin complement pathway dependent without involving C1q. J Immunol 174:6373-6380

26. Hu Y, Belke D, Suarez J et al (2005) Adenovirus-mediated overexpression of O-GlcNAcase improves contractile function in the diabetic heart. Circ Res 96:1006-1013

27. Sahn DJ, DeMaria A, Kisslo J, Weyman A (1978) Recommendations regarding quantitation in M-mode echocardiography: results of a survey of echocardiographic measurements. Circulation 58:1072-1083

28. Kenchaiah S, Pfeffer MA, St John Sutton JS et al (2004) Effect of antecedent systemic hypertension on subsequent left ventricular dilation after acute myocardial infarction (from the Survival and Ventricular Enlargement trial). Am J Cardiol 94:1-8

29. Tao L, Liu HR, Gao F et al (2005) Mechanical traumatic injury without circulatory shock causes cardiomyocyte apoptosis: role of reactive nitrogen and reactive oxygen species. Am J Physiol Heart Circ Physiol 288:H2811-H2818

30. Fleming TJ, Malek TR (1994) Multiple glycosylphosphatidylinositol-anchored Ly-6 molecules and transmembrane Ly-6E mediate inhibition of IL-2 production. J Immunol 153:1955-1962

31. Ramanadham S, Young J, Tenner TE Jr (1987) Prevention of streptozotocin-induced alterations in the rat heart by 3-O-methyl glucose and insulin treatments. J Cardiovasc Pharmacol 9:291297

32. Collard CD, Lekowski R, Jordan JE, Agah A, Stahl GL (1999) Complement activation following oxidative stress. Mol Immunol 36:941-948

33. Hart ML, Walsh MC, Stahl GL (2004) Initiation of complement activation following oxidative stress. In vitro and in vivo observations. Mol Immunol 41:165-171

34. Thiel S, Frederiksen PD, Jensenius JC (2006) Clinical manifestations of mannan-binding lectin deficiency. Mol Immunol 43: 86-96 